\title{
Variáveis significativas na avaliação da inteligência
}

\author{
Irai Cristina Boccato Alves \\ Instituto de Psicologia da Universidade de São Paulo
}

\begin{abstract}
Resumo
Algumas variáveis têm mostrado uma influência significativa nos resultados dos testes de inteligência, o que leva à necessidade de considerá-las e controlá-las em estudos com os mesmos. Entre elas podemos destacar o nível sócio-econômico, a idade, o sexo e o grau de escolaridade. O nível sócio-econômico mostrou ser relevante no Teste de Goodenough, nas Matrizes Progressivas Coloridas de Raven e na Escala de Maturidade Mental Colúmbia. Quanto à variável sexo, foram constatadas diferenças significantes entre os sexos no Teste de Goodenough, no Teste R-I e no D.70, mas não no Raven e Colúmbia. A idade cronológica sempre deve ser considerada na avaliação da inteligência de crianças, pois os resultados nos testes devem aumentar à medida que a criança cresce. $\mathrm{O}$ mesmo ocorre com relação à escolaridade, tanto quando se considera séries, como entre níveis de escolaridade.
\end{abstract}

Palavras-chave: avaliação psicológica, testes de inteligência, normas de testes.

\section{Significant variables in inteIligence evaluation}

\section{Summary}

Some variables have a significant influence in the results of intelligence tests, what. leads to the need of considering them and controlling them in studies with these tests. Among them we can highlight the socioeconomic level, the age, the sex and the school grade. The results showed differences between socioeconomic levels in the Test of Goodenough, in the Raven Coloured Progressive Matrices and in the Columbia. Mental Maturity Scale. Significant differences were found between mean scores of sexes in the Test of Goodenough, in the R-1 Test and in the D.70, but not in Raven and Columbia. The chronological age should always be considered in the assessment ofthe children's intelligence, because the results in the tests should increase as the child grows old. The same happens with the school grade, if we consider school grades or the school levels.

Key-words: psychological assessment, intelligence tests, norms of tests.

Os testes de inteligência são utilizados na avaliação psicológica para uma grande variedade de objetivos e contextos, sendo que estes estão constantemente se expandindo. Entre as áreas de utilização podem se destacar: a área ocupacional, na seleção e orientação profissional e na orientação de carreira; a área educacional para a avaliação e acompanhamento de crianças com problemas de aprendizagem, com deficiência intelectual ou superdotadas; a área clínica para realização de diagnósticos; a área hospitalar e a área judiciária.

Entretanto para que os testes de inteligência sejam empregados com segurança, bem como qualquer outro teste psicológico, é necessário o estabelecimento de normas apropriadas para a população em que os testes vão ser empregados. Conforme salientam Anastasi e Urbina (1997, p. 68) "qualquer norma é restrita à população normativa da qual foi derivada". Elas "não são absolutas, universais ou permanentes... Elas representam a realização no teste das pessoas que constituem a amostra de padronização". Essa amostra deve ser representativa da população da qual foi extraída. A representatividade da amostra deve considerar características relevantes sobre a variável que está sendo avaliada.

Dessa forma é importante lembrar que na avaliação de uma determinada característica psicológica não se deve empregar tabelas normativas provenientes de outros países, em função das 
diferenças sociais e culturais existentes. Muitas vezes as normas devem ser específicas para subgrupos da população, principalmente quando há diferenças consideráveis entre eles nos resultados de determinado teste. Os subgrupos podem variar quanto à idade, sexo, série escolar, região geográfica, nível sócio-econômico, etc.

O presente trabalho tem por objetivo mostrar como algumas variáveis têm uma influência significativa nos resultados dos testes de inteligência e a necessidade de considerá-las e controlá-las nos estudos sobre esse tema, bem como no estabelecimento de normas específicas para os subgrupos definidos por essas variáveis. Entre essas variáveis podemos destacar o nível sócioeconômico, o sexo, a idade, a série escolar e o grau de escolaridade.

Os dados apresentados neste trabalho foram baseados em pesquisas com testes de inteligência em que a autora participou e em alguns resultados da literatura, principalmente do Brasil.

A primeira variável a ser considerada é o nível sócio-econômico, que pode ser definido através da profissão e escolaridade dos pais, pela renda familiar, por algumas escalas relativas aos bens e eletrodomésticos que as famílias possuem, ou pelo tipo de escola freqüentada pela criança.

O nível sócio-econômico, avaliado pela profissão e escolaridade do pai, foi empregado numa pesquisa com o Teste de Inteligência através do Desenho da Figura Humana de Goodenough e sua revisão Goodenough-Harris em pré-escolares paulistanos, com idades variando de 4 anos e 3 meses a 6 anos e 9 meses (Alves, 1979 e 1981). Nesse teste pede-se à criança para desenhar um Homem e na revisão pede-se, além do desenho de um Homem, o Desenho de uma Mulher. Nessa pesquisa foram constatadas diferenças estatisticamente significantes entre as crianças classificadas como pertencentes à classe média-alta e as de classe média-baixa, tanto no Teste de Goodenough, quanto na sua revisão, nos desenhos do Homem e da Mulher. Os resultados dessa comparação podem ser observados na Tabela 1, que apresenta as médias do Teste de Goodenough e dos desenhos do Homem e da Mulher da escala revisada. Como pode ser constatado os resultados das crianças da classe média-alta foram superiores aos da classe média-baixa.

Tabela 1. Comparação entre os níveis socioeconômicos no Teste de Goodenough e Goodenough-Harris

\begin{tabular}{|c|c|c|c|c|c|c|}
\hline & \multicolumn{2}{|c|}{ Goodnough } & \multicolumn{2}{c|}{ Homem } & \multicolumn{2}{c|}{ Mulher } \\
\hline Idade & M. alta & M. baixa & M. alta & M. baixa & M. alta & M. baixa \\
\hline 4,5 & 8,70 & 6,72 & 9,57 & 7,32 & 9,27 & 6,75 \\
\hline 5 & 11,30 & 7,80 & 13,80 & 8,82 & 13,37 & 9,30 \\
\hline 5,5 & 12,67 & 8,70 & 14,30 & 10,15 & 15,77 & 9,75 \\
\hline 6 & 14,55 & 11,22 & 16,52 & 12,60 & 16,20 & 12,45 \\
\hline 6,5 & 16,65 & 13,60 & 18,65 & 15,55 & 18,50 & 15,50 \\
\hline
\end{tabular}

Na pesquisa de padronização da Escala de Maturidade Mental Colúmbia (Alves e Duarte, 1993) a amostra foi dividida em função do tipo de escola freqüentada pelas crianças, mantendo-se na amostra as mesmas proporções de crianças matriculadas nos três tipos de escolas: particulares, 
municipais e estaduais. Esse critério foi adotado porque geralmente as crianças de escolas públicas (estaduais e municipais) apresentam nível sócio-econômico mais baixo do que as das escolas particulares e com o sorteio das escolas dentro desses três tipos se poderia conseguir melhor representatividade em relação a essa variável. A faixa etária das crianças variou entre 3 anos e 6 meses e 9 anos e 11 meses. Este teste está dividido em níveis, que correspondem aos itens mais apropriados para a faixa etária da criança, sendo que há uma sobreposição parcial dos itens.

Os resultados indicaram que não havia diferenças entre os resultados médios das crianças de escolas municipais e estaduais, ou seja, entre as escolas públicas. No entanto foram encontradas diferenças significantes entre os resultados das crianças das escolas particulares e das escolas públicas, apontando para a necessidade de estabelecer normas separadas para os dois tipos de escolas.

Também no Teste das Matrizes Progressivas Coloridas de Raven (Alves, Angelini, Custódio, Duarte e Duarte, 1995a) foi empregado o mesmo critério de seleção de amostra entre crianças de escolas públicas e particulares, para crianças entre 4 anos e 9 meses e 11 anos e 9 meses. Nesse caso também houve diferenças significantes entre os resultados médios das crianças de escolas públicas e de particulares, mas não entre as dos dois tipos de escolas públicas (municipais e estaduais). Para este teste também foram construídas tabelas de normas separadas para cada um dos dois tipos de escolas (Alves, Angelini, Custódio, Duarte e Duarte, 1995b).

Tanto no caso da Escala de Maturidade Mental Colúmbia, como no das Matrizes Progressivas Coloridas as crianças de escolas particulares obtiveram resultados mais altos.

Quanto à variável sexo, há uma certa variação nos resultados, conforme o teste estudado e, às vezes, conforme a faixa etária.

Na pesquisa com o Teste Goodenough e sua revisão Goodenough-Harris com pré-escolares (Alves, 1979 e 1981) foram encontradas diferenças significantes quanto ao sexo para o Teste de Goodenough e apenas para o Desenho da Mulher na revisão. Foi verificado que as meninas tiveram resultados mais altos nessas duas escalas.

Na literatura com esse teste alguns estudos relatam superioridade das meninas, outros, dos meninos e alguns não apontam diferenças. A própria autora, Goodenough (1926) encontrou resultados maiores das meninas, atribuindo essa diferença ao método usado para a estandardização dos itens e a diferenças entre os sexos no progresso escolar. Em seus estudos normativos Harris (1963) também encontrou diferenças, tanto no Desenho do Homem como no da Mulher, na escala revisada. Foi observado que as diferenças entre os desenhos são maiores nas idades menores e diminuem para as crianças de 6 anos e meio, nas duas avaliações do Desenho do Homem, mas não no Desenho da Mulher. Desta forma as diferenças podem também estar associadas à idade, e como existem resultados muito contraditórios nas pesquisas, pode ser uma variável que esteja sofrendo influência de fatores culturais. Entretanto os resultados maiores das meninas no Desenho da Mulher podem estar relacionados a uma maior identificação sexual das meninas com a figura feminina e com seu papel sexual, o que seria refletido no Desenho do próprio sexo.

Por outro lado, na pesquisa de padronização da Escala de Maturidade Colúmbia (Alves e Duarte, 1993) não foram constatadas diferenças entre os sexos, em cada um dos níveis do teste. Por se tratar de um teste não verbal que avalia a capacidade geral de raciocínio é provável que o resultado no teste não seja influenciado pelo sexo do sujeito.

O mesmo ocorreu na pesquisa de padronização do Teste das Matrizes Coloridas de Raven 
(Alves, Angelini, Custódio e Duarte, 1988), onde não foram constatadas diferenças entre os sexos, a não ser para as crianças de 11 anos e 11 anos e meio.

Resultados semelhantes foram obtidos por Meio (1998) ao comparar os resultados de meninos e meninas, de 10 e de 13 anos, na Escala Verbal Brasileira do WISC adaptada por Lemgruber e Paine (1981) e na Escala Verbal do WISC traduzida por Poppovic (Wechsler, 1964). Não foram verificadas diferenças entre os sexos em todos os subtestes verbais, exceto para o subteste de Compreensão na a escala traduzida para a idade de 10 anos e para a amostra total. Nesse caso as meninas obtiveram resultados mais altos, o que poderia estar mostrando uma maior maturidade social das meninas aos 10 anos, mas que não aparece mais aos 13 anos.

Já em pesquisas brasileiras realizadas com adultos pode-se citar uma feita com o R-I e outra com o D.70.

Na pesquisa realizada com adultos de três níveis de escolaridade com o Teste R-I (Alves, Colosio e Ruivo, 1992) foram constatadas diferenças estatisticamente significantes entre os sexos, nos três níveis estudados, sendo que os sujeitos do sexo masculino obtiveram resultados mais altos. Entretanto como essa pesquisa foi composta por uma amostra de candidatos a emprego em uma empresa não é possível garantir que seja representativa da população, principalmente, porque a maioria dos cargos para o sexo feminino eram atividades de baixo prestígio social, o que de certa forma poderia explicar os resultados obtidos pelo sexo feminino.

Na pesquisa de padronização do D.70 também foram encontradas diferenças entre os sexos, tanto no grupo de escolares do $2^{\circ}$ grau, como no de adultos com escolaridade de $2^{\circ}$ grau e para adultos do $3^{\circ}$ grau, o que levou à construção de tabelas normativas para cada sexo. Nesse caso também o sexo masculino obteve resultados mais altos (Centre de Psychologie Appliquée, 1988).

Uma variável cujo efeito é esperado e que sempre deve ser considerado em testes de inteligência para crianças é a idade cronológica, uma vez que os resultados devem aumentar à medida que a criança cresce.

O aumento no número médio de pontos com a idade foi observada no Teste Goodenough e sua revisão Goodenough-Harris, na Escala de Maturidade Mental Colúmbia e nas Matrizes Progressivas Coloridas de Raven. Dessa forma os testes devem apresentar normas para cada idade, ou até mesmo para cada meio ano, em função de diferenças encontradas para o teste em questão.

No entanto com adultos a idade é uma variável mais complexa, isto porque se supõe um patamar até onde a inteligência aumenta em função da idade. Durante algum tempo os autores discutiram se a inteligência parava de crescer aos 16 ou aos 18 anos, que foram os limites usados na Escala Stanford-Binet de 1937 e 1960 (Anastasi, 1977). Isto se justificava porque os resultados médios nos testes deixam de aumentar significativamente nessa idade. Cunha (1974, p.101) através de um levantamento da literatura afirma que "a curva do crescimento intelectual do homem tem aceleração positiva na infância e na pré-adolescência; esta aceleração decresce, sensivelmente na adolescência, tornando-se nula na idade adulta. Da idade madura para a velhice, a curva apresentaria um declínio significante". Na pesquisa de padronização do W AIS foi observado que o resultado máximo ocorria entre 20 e 30 anos, com um leve declínio até os 60 anos, acentuando-se a partir dessa idade (Anastasi, 1977). Esse resultado também foi obtido por Weil e Nick (s / d), na pesquisa sobre o potencial de inteligência do brasileiro, realizada com o INV. Resultados semelhantes foram apresentados por Raven, Court e Raven em 1985, ou seja, considerava-se que a capacidade intelectual declinava a partir dos 25 anos de idade. 
Essa conclusão é discutida por Matarazzo (1976), em função do papel da educação, pois quando se faz um estudo transversal, os indivíduos mais velhos viveram em um meio educacional e tiveram experiências muito diferentes dos mais jovens, bem como em média tiveram menos anos de escolaridade. Isto leva à impressão de um declínio com a idade, que é aparente, uma vez que os estudos longitudinais mostraram que a inteligência do adulto continua a aumentar modestamente até os 50 anos e então mostra um pequeno declínio acima desta idade. A partir desses resultados Matarazzo levanta o problema da atualização constante das normas dos testes para corrigir essas possíveis distorções.

Em um trabalho mais recente Raven, Raven e Comi (1991) mostram que ao comparar estudos realizados em diferentes épocas, mas relativas a pessoas nascidas nos mesmos anos, mas que tivessem diferentes idades nos anos em que os estudos foram realizados, apresentavam resultados semelhantes, independentemente do ano em que foram testados. Isto mostra que não há um declínio com a idade, mas que o resultado está relacionado ao ano de nascimento, o que faz com que pessoas nascidas há mais tempo tenham resultados menores no Teste de Raven, mostrando a influência das variáveis culturais e nutricionais no sentido de aumentar os resultados médios no teste com o passar dos anos.

Na pesquisa com o R-I (Alves, Colosio e Ruivo) também foi constatado um declínio no número de pontos com o aumento da idade, conforme indicavam as pesquisas mencionadas anteriormente. Porém, ao se fazer um levantamento da escolaridade em relação à idade, verifica-se que na amostra há uma maior freqüência de sujeitos com escolaridade primária entre 30 e 45 anos, com nível ginasial entre 20 e 35 anos e com $2^{\circ}$ grau entre 20 e 30. Esses dados mostram que os sujeitos de mais idade possuem um grau de escolaridade menor, o que parece estar contribuindo para esse resultado, bem como estão de acordo com as afirmações de Matarazzo (1976) e de Raven, Raven e Court (1991).

Outra variável a ser discutida neste trabalho é a escolaridade, seja ela em termos da série escolar da criança ou de nível de escolaridade do adulto.

Na pesquisa de padronização do Teste das Matrizes Progressivas Coloridas de Raven também foi feito um estudo em relação à série escolar em que a criança está e foram constatadas diferenças significantes nos resultados médios entre as séries escolares, com um crescimento nos pontos à medida que a série aumenta (Alves et al, 1988). No entanto, como há uma forte associação entre a série e a idade, é preferível empregar normas por idade do que por série. As normas por série seriam de maior interesse para tentar situar a maturidade intelectual de uma criança atrasada em termos de escolaridade.

Quanto ao grau de escolaridade no adulto muitas pesquisas mostram um crescimento no número de pontos nos testes conforme aumenta o nível de escolaridade.

No R-I observou-se que o resultado médio aumentava em função do aumento da escolaridade e esse resultado apareceu para os dois sexos. A mesma conclusão pode ser tirada se forem comparadas as médias obtidas no D.70 por sujeitos com escolaridade de $2^{\circ}$ e de $3^{\circ}$ graus.

A partir dos aspectos apresentados alerta-se para a necessidade dos pesquisadores investigarem a influência das variáveis aqui apresentadas antes de construírem normas para os testes, bem como de construírem tabelas separadas, caso essa influência seja confirmada. Ao mesmo tempo alerta-se ao profissional que vai fazer uma avaliação a verificar se no Manual do teste ou em pesquisas publicadas posteriormente essas variáveis foram consideradas e consultar tabelas 
apropriadas levando em conta essas variáveis.

\section{Referências}

Alves, I. C. B. (1979). O Teste Goodenough-Harris em uma população pré-escolar paulistana. Dissertação de Mestrado - Instituto de Psicologia da Universidade de São Paulo, São Paulo.

Alves, I. C. B. (1981). O Teste Goodenough-Harris em Pré-Escolares Paulistanos. Boletim de Psicologia, XXXIII, 80, 40-52.

Alves, I. C. B.; Angelini, A. L.; Custódio, E. M.; Duarte, W. F. (1988). O Teste das Matrizes Progressivas Coloridas de Raven: "Um estudo das variáveis idade, sexo e escolaridade", Resumos da XV/l1 Reunião da Sociedade de Psicologia de Ribeirão Preto, 18, 163.

Alves, I. C. B.; Angelini, A. L.; Custódio, E. M.; Duarte, W. F. E Duarte, 1. L. M. (I 995a). O Teste das Matrizes Progressivas Coloridas de Raven: a Influência do tipo de escola, /II Congresso Interno do Instituto de Psicologia. Resumos, 55.

Alves, I. C. B.; Angelini, A. L.; Custódio, E. M.; Duarte, W. F. E Duarte, J. L. M. (1995b). O Teste das Matrizes Progressivas Coloridas de Raven: Normas Complementares em função do Tipo de Escola, 1/1 Congresso Interno do Instituto de Psicologia. Resumos, 56.

Alves, I. C. B.; Colosio, R. e Ruivo, R. 1. (1992). O Teste R-I: Um estudo das variáveis idade, sexo e escolaridade. Anais da 44" Reunião Anual da Sociedade Brasileira para o Progresso da Ciência, 44, 904.

Alves, I. C. B. e Duarte, J. L. M. (1993). Padronização Brasileira da Escala de Maturidade Mental Colúmbia. In Burgemeister, B. B.; Blum, L. H. e Lorge, I. - Escala de Maturidade Mental Colúmbia - 3" Edição. Manual para Aplicação e Interpretação. São Paulo: Casa do Psicólogo.

Anastasi, A. (1977). Testes Psicológicos. São Paulo: EPU.

Anastasi, A. e Urbina, S. (1997). Psychological Testing. Upper Sadle River, N. J.: Prentice Hall, 7th ed.

Centre de Psychologie Appliquée (1988). Teste D.70 : Manual. São Paulo: Casa do Psicólogo.

Cunha, S. E. (1974). A Psicometria da Inteligência e a dimensão idade. Arquivos Brasileiros de Psicologia Aplicada, 26, 3, 100-110.

Goodenough, F. L. (1926). Measurement of intelligence by drawings. New York: World Book Co.

Harris. D. B. (1963). Children's drawings as measure of intellectual maturity. New York: Harcourt, Brace \& World.

Lemgruber, V. e Paine, P. A. (1981). A Adaptação Brasileira da Escala Verbal do WISC. Arquivos Brasileiros de Psicologia, 33, 1-2,32-56.

Matarazzo, J. (1976). Wechsler. Medida e Avaliação da Inteligência do Adulto. São Paulo: Manole.

Melo, M. G. (1998). Estudo Comparativo da Escala Verbal do WISC Adaptada por Paine e Lemgruber com a Escala Traduzida por Poppovic. Dissertação de Mestrado - Instituto de Psicologia da Universidade de São Paulo, São Paulo.

Raven, J. C.; Court, 1. H. e Raven, J. (1985). Manual for Raven's Progressive Matrices and Vocabulary Scales. Section l. General Overvie. London: H. K. Lewis \& Co.

Raven, 1.; Raven, J. C. e Court, 1. H. (1991). Manual for Raven's Progressive Matrices and Vocabulary Seales. Section l. General Overview. Oxford: Oxford Psychologists Press.

Wechsler, D. (1964). Manual da Escala de Inteligência Wechsler para Crianças. Rio de Janeiro: CEPA.

Weil, P. e Nick, E. (s/d). O Potencial da Inteligência do Brasileiro. Rio de Janeiro: CEPA. 